\title{
Functional impact of colorectal cancer-associated mutations in the transcription factor E2F4
}

\author{
MARIE-CHRISTINE PAQUIN, CAROLINE LEBLANC*, ETIENNE LEMIEUX*, \\ BENJAMIN BIAN and NATHALIE RIVARD
}

\begin{abstract}
Department of Anatomy and Cellular Biology, Cancer Research Pavillon, Faculty of Medicine and Health Sciences, Université de Sherbrooke, Sherbrooke, Québec J1E4K8, Canada
\end{abstract}

Received August 7, 2013; Accepted September 9, 2013

DOI: $10.3892 /$ ijo.2013.2131

\begin{abstract}
The transcription factor E2F4 plays a critical role in cell cycle progression of normal and cancerous intestinal epithelial cells. Contrary to other E2Fs, the coding region of the E2F4 gene contains a longer spacer segment of a CAG trinucleotide repeat sequence encoding 13 consecutive serine residues, which is highly vulnerable to frameshift mutations in situations of genetic instability. Mutations in this region of the $E 2 F 4$ gene have been observed in colorectal tumors with microsatellite instability. However, the effect of these changes on its function in colorectal cancer cells is currently unknown. We generated E2F4(CAG) $)_{12}$ and E2F4(CAG) $)_{14}$ mutants and compared their activity to the E2F4 wild-type, E2F4(CAG) $)_{13}$. Luciferase assays with the thymidine kinase-luc reporter gene revealed that the mutants were more transcriptionally active than wild-type E2F4. The mechanism of increased activity of E2F4 was primarily related to protein stability, due to a significantly enhanced half-life of E2F4 mutants comparatively to that of wild-type E2F4. However, the association with the pocket protein p130/RBL2 did not account for this increased protein stability. Sequencing analysis of the endogenous E2F4 gene in a series of colorectal cancer cell lines showed that the microsatellite-unstable cell line SW48 exhibited a serine deletion in this gene. Accordingly, E2F4 half-life was much more elevated in SW48 cells in comparison to Caco-2/15, a microsatellite-stable cell line. Notably, in soft-agar assays, both mutants more potently increased anchorage-independent growth in comparison to wild-type E2F4. In conclusion, our data demonstrate that cancer-associated E2F4 mutations
\end{abstract}

Correspondence to: Dr Nathalie Rivard, Department of Anatomy and Cellular Biology, Cancer Research Pavillon, Faculty of Medicine and Health Sciences, Université de Sherbrooke, 3201 Jean Mignault, Sherbrooke, Québec J1E4K8, Canada

E-mail: nathalie.rivard@usherbrooke.ca

*Contributed equally

Key words: E2F4, colorectal cancer, cell cycle, microsatellite instability, cancer growth enhance the capacity of colorectal cancer cells to grow without anchorage, thereby contributing to tumor progression.

\section{Introduction}

Colorectal cancer (CRC) represents one of the major causes of cancer-associated deaths in North America and worldwide (1). Since the entire intestinal epithelium is in a constant state of renewal (2), such turnover involves a countless number of cell divisions, resulting in a non-negligible risk of genetic alterations. This rapid rate of intestinal epithelial renewal as well as exposure to toxic substances, notably through food ingestion, may explain the high prevalence of CRC. CRC is a heterogeneous disease displaying distinct molecular signatures and distinct pathological features. There are at least three major molecular pathways to $\mathrm{CRC}$ including the predominant chromosomal instability (CIN) pathway, the $\mathrm{CpG}$ island methylator phenotype (CIMP) pathway which is the other major pathway to sporadic CRC and includes sporadic microsatellite instability (MSI) high cancers and finally the pure MSI pathway resulting from germline mutation in a DNA mismatch repair (MMR) gene $(1,3,4)$.

One of the most important cellular barriers to cancer development is the retinoblastoma tumor suppressor ( $\mathrm{pRB}$ ) pathway, which is inactivated in a wide range of human tumors and controls cell cycle progression via repression of the E2F/DP transcription factor family. Indeed, when hypophosphorylated, $\mathrm{Rb}$ proteins bind to $\mathrm{E} 2 \mathrm{~F} / \mathrm{DP}$ dimers, preventing transactivation of their target genes $(5,6)$. The E2F/DP transcription factor family currently numbers 8 members (E2F1-8). DNA microarray analysis reveals unique sets of target promoters among E2F family members suggesting that each protein may have a unique role in the cell cycle (7). Among E2F transcriptional targets are cyclins, cdks, checkpoints regulators, DNA repair and replication proteins.

We previously reported that E2F4 silencing in normal human intestinal epithelial cells markedly reduced the expression of many cell cycle regulatory genes including thymidine kinase, $c$-myc, cdc6 and cyclin Al, slowing their proliferation rate (8). Moreover, double staining experiments in vivo and in vitro revealed that intestinal crypt epithelial cells which expressed high levels of nuclear E2F4, were positive for Ki67 (9) and cyclin A1 (8). We have also provided evidence 
that E2F4 expression is important for growth of colon cancer cell lines. Indeed, reduction of E2F4 protein expression significantly slowed the proliferation rate and soft-agar growth of colon cancer cell lines.

Interestingly, in contrast to other E2F encoding genes, the $\mathrm{E} 2 \mathrm{~F}$-4 gene exhibits frequent tumor-specific mutations at a coding region of trinucleotide microsatellite (CAG)n in a subset of human sporadic CRC with high-frequency MSI (MSI-H) (10-13). Importantly, this trinucleotide repeat (13 consecutive serines) is localized in the transactivation domain (14). The two most frequent mutations observed are the deletion or the addition of one trinucleotide CAG in the E2F4 serine stretch $\left.\left[\mathrm{E} 2 \mathrm{~F} 4(\mathrm{Ser})_{12} \text { and E2F4(Ser) }\right)_{14}\right](10,12)$. However, the functional impact of these molecular alterations on E2F4 expression, activity and function in colorectal cancer cells remains to be demonstrated.

\section{Materials and methods}

Materials and antibodies. Antibodies against E2F4 (C-20), p130 (C-20), DP-2 (C-20), and HA-probe (F-7) were all purchased from Santa Cruz Biotechnology Inc. (Santa Cruz, CA, USA). $\beta$-actin monoclonal antibody (clone C-4) was obtained from Millipore (Billerica, MA, USA). Cycloheximide was purchased from Calbiochem (San Diego, CA, USA). All other materials were from Sigma-Aldrich (Oakville, ON, Canada) unless stated otherwise.

E2F4, DP2 and p130 expression vectors. The expression vectors (pCDNAneo3) encoding for E2F4 and p130 were obtained from Dr C. Sardet (14) (Institut de Génétique Moléculaire, Montpellier, France). The full length E2F4 cDNA was subcloned into a pLVX-Tight-Puro (Clontech, CA, USA) expression vector. PCR was performed to insert the HA-tag using oligonucleotides containing the HA-tag and a KOZAK sequence: 5'-A GAC TAG GAT CC C ACC ATG TAT GAT GTT CCT GAT TAT GCT AGC CTC CCG GCG GAG GCC GGG CCA CAG GCG CCG-3' and 5'-TCT GTA CTC GAG TCA GAG GTT GAG AAC AGG CAC ATC AAA GAG GTC-3'. PCR products were next digested and ligated in BamHI/EcoRI-digested pLVX-Tight-Puro vector. E2F4 mutants $(\mathrm{Ser})_{12}$ and $(\mathrm{Ser})_{14}$ were obtained by site-directed mutagenesis using the following primers, respectively: 5'-CTG GAC AGC AGC AGC AGC AGC AGC AGC AGC AGC AGC AGC AGC AAC AGT AAC-3' and 5'-GTT ACT GTT GCT GCT GCT GCT GCT GCT GCT GCT GCT GCT GCT GCT GTC CAG-3'; 5'-CTG GAC AGC AGC AGC AGC AGC AGC AGC AGC AGC AGC AGC AGC AGC AGC AAC AGT AAC-3' and 5'-GTT ACT GTT GCT GCT GCT GCT GCT GCT GCT GCT GCT GCT GCT GCT GCT GCT GTC CAG-3'. pBABE-Puro HA-wtE2F4, HA-E2F4(Ser) $)_{12}$ and $\mathrm{HA}-\mathrm{E} 2 \mathrm{~F} 4(\mathrm{Ser})_{14}$ vectors were obtained by subcloning from pLVX-Tight-Puro vectors (BamHI/EcoRI). The pCMVHA-DP2 expression vector was obtained from Dr J.A. Lees (15) (Department of Biology, Massachusetts Institute of Technology, Cambridge, MA, USA).

Cell culture. Human embryonic kidney 293 cells and all colorectal cancer cell lines were obtained from ATCC (Manassas, VA, USA). 293T cells were cultured in Dulbecco's modified Eagle's medium (DMEM; Invitrogen, Burlington, ON, Canada) containing 10\% FBS supplemented with $2 \mathrm{mM}$ glutamine, $10 \mathrm{mM}$ HEPES, $0.5 \mathrm{IU} / \mathrm{ml}$ penicillin and $50 \mu \mathrm{g} /$ $\mathrm{ml}$ streptomycin (all obtained from Wisent, St-Bruno, QC, Canada). The human colorectal cancer cells were cultured in the following media: HT-29 and HCT116 in McCoy's 5A medium; DLD-1 and COLO205 in RPMI-1640 medium; LoVo in F-12K medium and Caco-2/15 in DMEM medium. All of the above media were supplemented with $10 \%$ FBS, $2 \mathrm{mM}$ glutamine, $10 \mathrm{mM}$ HEPES, $0.5 \mathrm{IU} / \mathrm{ml}$ penicillin and $50 \mu \mathrm{g} / \mathrm{ml}$ streptomycin. Non-immortalized human intestinal epithelial cells (HIEC) were isolated by Perreault and Beaulieu (16) and cultured in Opti-MEM (Invitrogen) medium supplemented with $2 \mathrm{mM}$ glutamine (Invitrogen), $5 \%$ fetal bovine serum (FBS), $10 \mathrm{mM}$ HEPES, $0.5 \mathrm{IU} / \mathrm{ml}$ penicillin, $50 \mu \mathrm{g} / \mathrm{ml}$ streptomycin and $0.2 \mathrm{IU} / \mathrm{ml}$ insulin (Connaught Novo Laboratories, Willowdale, Canada). HIEC, originally generated from normal human fetal small intestine at mid-gestation, express typical features of the lower adult crypt region and are unable to differentiate (16).

Retroviral infections. E2F4 retroviruses were produced by co-transfecting pBabe-puro HA-wtE2F4, $(\mathrm{Ser})_{12}$ or (Ser) ${ }_{14}$ constructs with the pVPack vector system (Agilent Technologies, Mississauga, ON, Canada) in 293T cells using Lipofectamine 2000 (Invitrogen) according to the manufacturer's recommendations. Viral supernatants were harvested $48 \mathrm{~h}$ following transfection, filtered through a $0.45-\mu \mathrm{m}$ filter and cells were infected following the instructions of Agilent Technologies.

Protein extraction and immunoblotting. Cells were washed twice with ice-cold PBS, then lysed in Triton lysis buffer [1\% Triton X-100, $50 \mathrm{mM}$ Tris- $\mathrm{HCl} \mathrm{pH} 7.5,100 \mathrm{mM} \mathrm{NaCl}$, $5 \mathrm{mM}$ EDTA, 5\% glycerol, $40 \mathrm{mM} \beta$-glycerophosphate, $50 \mathrm{mM} \mathrm{NaF}, 200 \mu \mathrm{M}$ orthovanadate, $5 \%$ pepstatin, $5 \%$ aprotinin, $5 \%$ leupeptin and 5\% phenylmethylsulfonyl fluoride (PMSF)] for 30 min under light agitation. Lysates were then cleared by centrifugation $(15,000 \mathrm{~g}, 10 \mathrm{~min})$ and $4 \mathrm{X}$ Laemmli buffer $(2.3 \%$ SDS, $10 \%$ glycerol, $0.005 \%$ bromophenol blue and $5 \% \quad \beta$-mercaptoethanol) was added to supernatants for gel analysis. Whole cell extracts were separated on $10 \%$ SDS-PAGE gels and then electro-transferred onto polyvinylidene fluoride (PVDF) membranes (Perkin-Elmer, Montréal, Québec, Canada). Membranes were blocked for $1 \mathrm{~h}$ at $20^{\circ} \mathrm{C}$ using $0.05 \%$ Tween/PBS containing $5 \%$ non-fat dry milk then incubated overnight in primary antibodies diluted in blocking solution. Membranes were next incubated with horseradish peroxidase-conjugated goat anti-mouse or antirabbit IgG (GE Healthcare, Baie d'Urfé, Canada) in blocking solution for $1 \mathrm{~h}$. The blots were visualized using homemade ECL (Tris- $\mathrm{HCl} 100 \mathrm{mM}$ pH 8.5, $1.25 \mathrm{mM}$ luminol, $225 \mu \mathrm{M}$ coumaric acid and $2.9 \mathrm{mM} \mathrm{H}_{2} \mathrm{O}_{2}$ ). Protein concentrations were measured using the BCA procedure (Thermo Scientific, Waltham, MA, USA) as described by the manufacturer, with bovine serum albumin (BSA) as standard.

Transfection, stability, luciferase and coimmunoprecipitation assays. 293T cells were co-transfected with pLVX-Tight-Puro empty vector, HA-wtE2F4, HA-E2F4(Ser) ${ }_{12}$ or HA-E2F4(Ser) ${ }_{14}$ 
and pLVX Tet-Off Advanced using Lipofectamine 2000 (Invitrogen) according to the manufacturer's protocol. Cells were cultured in absence of tetracycline to allow E2F4 expression. For stability assays, cells were transfected for $24 \mathrm{~h}$ then treated with cycloheximide for the indicated times. For luciferase assays, 293T cells were also transfected with pE2F-TA-Luc luciferase reporter vector (Clontech), pRL-SV40 Renilla luciferase reporter (Promega, Madison, WI, USA) and pCMV-HA-DP2 vector. Forty-eight hours following transfection, luciferase activity was measured using the DualLuciferase ${ }^{\circledR}$ Assay Reporter System (Promega) according to the manufacturer's protocol. Relative E2F4 luciferase activity was normalized using the Renilla reporter to account for transfection efficiency. For co-immunoprecipitation assays, cells were transfected for $48 \mathrm{~h}$ and lysed with Triton lysis buffer. Cleared lysates were incubated with anti-E2F4 antibody $\left(3 \mathrm{~h}, 4^{\circ} \mathrm{C}\right)$, after which protein A Sepharose CL-4B beads (GE Healthcare) were added for an additional hour. Immunocomplexes were washed three times with Triton X-100 lysis buffer then eluted with Laemmli buffer and loaded on SDS-PAGE gels. Extensive protocols of 293T cells transfection and luciferase assays are available upon request.

Characterization of the number of CAG repeats in the E2F4 microsatellite using capillary electrophoresis. Genomic DNAs (gDNAs) were isolated from the human colorectal cancer cells Caco-2/15, COLO205, HT-29, LoVo, DLD-1, HCT116 et SW48 as well as from normal HIEC using the Spin Doctor Solution Set (Gerard Biotech, Oxford, OH, USA) according to the manufacturer's protocol. The E2F4 microsatellite was amplified by PCR from obtained gDNAs and PCR products were analyzed by capillary electrophoresis by the Laboratoire de Génomique Fonctionnelle de l'Université de Sherbrooke in order to determine the number of CAG repeats in the E2F4 microsatellite in each of the intestinal cell lines using control plasmid DNA with 12,13 or 14 CAG repeats [pLVX-TightPuro E2F4 (Ser) ${ }_{12}$, wtE2F4 and E2F4(Ser) $)_{14}$, respectively]. The Agilent 2100 Bioanalyzer (Agilent Technologies) and the LabChip ${ }^{\circledR} 90$ (Caliper, Hopkinton, MA, USA) were used according to the manufacturer's recommendations.

RNA extraction and RT-PCR analysis. Total RNA was isolated with the RNeasy mini kit (Qiagen, Mississauga, ON, Canada). RT-PCR was performed using avian myeloblastosis virus reverse transcriptase (Roche Diagnostics) and conventional PCR analysis was conducted using Taq DNA polymerase according to the manufacturer's instructions (Qiagen). Realtime PCR analyses were performed with a LightCycler apparatus (Roche Diagnostics). Experiments were executed and analyzed with the LightCycler software 4.0 according to the manufacturer's recommendations. Synthesis of doublestranded DNA during PCR cycles was monitored with SYBR Green I (QuantiTect SYBR Green PCR kit; Qiagen). All samples were processed in triplicate. E2F4 expression was quantified relative to $\beta 2$-microglobulin expression. A standard calibration curve was prepared for each gene by using serial dilutions of the calibrator sample, and crossing point values were plotted vs. the log of the relative concentration of each dilution. This standard curve was used to correct for differences in PCR efficiencies. Oligonucleotide primers used for
DNA amplification were synthesized by Integrated DNA Technologies (San Diego, CA, USA). Primer sequences are available upon request.

Soft agar assays. RPMI medium (Wisent, QC, Canada) was complemented with $20 \%$ FBS, $4 \mathrm{mM}$ glutamine, $20 \mathrm{mM}$ HEPES, $1 \mathrm{IU} / \mathrm{ml}$ penicillin and $100 \mu \mathrm{g} / \mathrm{ml}$ streptomycin. This pre-warmed medium was mixed $1: 1(\mathrm{v} / \mathrm{v})$ with autoclaved $1.4 \%$ agarose type VII maintained at $\sim 42^{\circ} \mathrm{C}$ and 6 -well dishes were pre-coated with $1.5 \mathrm{ml} /$ well. A total of 30,000 cells/ well were added to the RPMI/agarose mixture. Plates were allowed to solidify then placed at $37^{\circ} \mathrm{C}$ under $5 \% \mathrm{CO}_{2}$. Fresh RPMI supplemented with $10 \%$ FBS was added on the surface of the agarose every 2-3 days. After 2-3 weeks, colonies were stained by adding $1 \mathrm{ml}$ of PBS containing $0.5 \mathrm{mg} / \mathrm{ml}$ MTT to each well and incubated $2 \mathrm{~h}$ at $37^{\circ} \mathrm{C}$ and $5 \% \mathrm{CO}_{2}$. Images were acquired using an AlphaImager camera (Alpha Innotech Corp.). Colonies were counted and their size assessed using ImageJ software.

Data presentation. Assays were performed in triplicate. Typical western blots shown are representative of three independent experiments. Densitometric analyses were performed using ImageJ software.

\section{Results}

Addition or withdrawal of a single serine residue in the E2F4 microsatellite increases E2F4 transcriptional activity and protein expression. To determine the impact of serine stretchassociated mutations on E2F4 function, both mutant forms of E2F4 reported to be frequently observed in CRC were generated, namely E2F4(Ser $)_{12}$ and E2F4(Ser) $)_{14}$. These mutants as well as the wild-type form of E2F4 were then cotransfected along with thymidine kinase luciferase reporter gene. Previous studies have reported that the gene encoding for this protein contains E2F4-responsive elements in their promoters (17-19) and our previous studies have shown that E2F4 silencing in HIEC markedly reduced thymidine kinase mRNA levels (8). As shown in Fig. 1A, both E2F4(Ser) ${ }_{12}$ and E2F4(Ser) ${ }_{14}$ mutants exhibited significant enhanced transcriptional activities relative to thymidine kinase gene compared with wtE2F4. The increased transcriptional activity of mutants versus wildtype E2F4 prompted us to verify their mRNA and protein expression by quantitative PCR and western blot analyses respectively. As shown in Fig. 1B, protein levels of E2F4(Ser) ${ }_{12}$ and $\mathrm{E} 2 \mathrm{~F} 4(\mathrm{Ser})_{14}$ mutants were consistently higher than levels of wild-type E2F4. Of note however, these increased protein expression levels of mutants could not be attributed to an increase in mRNA levels which were similar to those of wildtype E2F4 (Fig. 1C).

Colorectal cancer-associated mutations increase E2F4 protein stability. Since increased protein but not mRNA levels were observed for $\mathrm{E} 2 \mathrm{~F} 4(\mathrm{Ser})_{12}$ and $\mathrm{E} 2 \mathrm{~F} 4(\mathrm{Ser})_{14}$ mutants, this suggests that colorectal cancer-associated mutations may affect protein stability of E2F4. Therefore, 293T cells were transiently transfected with either wild-type E2F4, E2F4(Ser) ${ }_{12}$ mutant or E2F4(Ser) ${ }_{14}$ mutant and then treated with cycloheximide to inhibit protein synthesis. Thereafter, cells were lyzed 
A

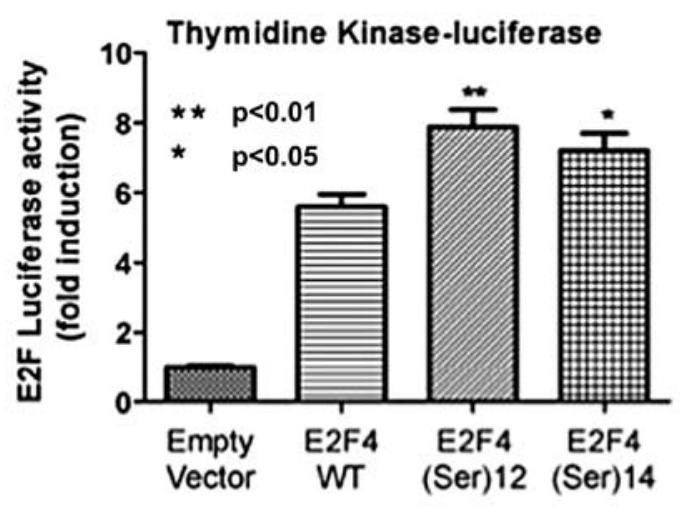

B

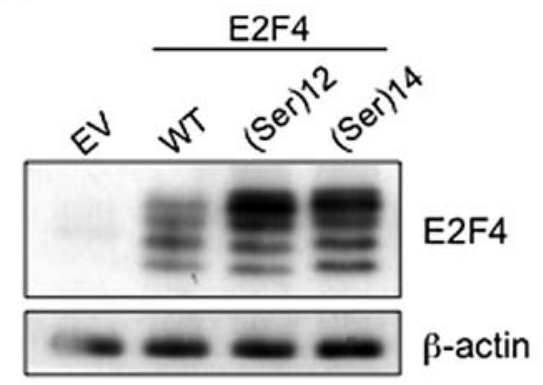

C

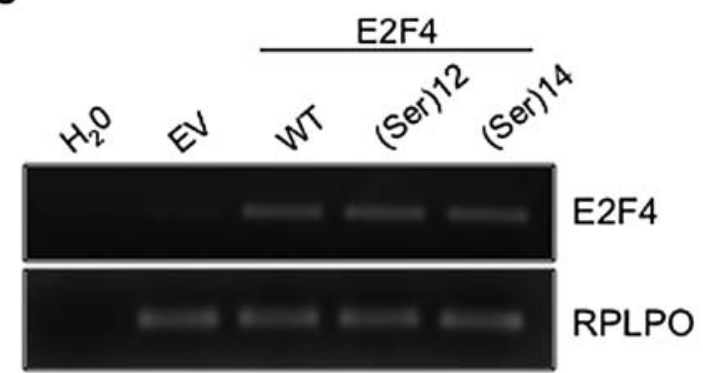

Figure 1. Addition or withdrawal of a single serine residue in E2F4 microsatellite increases its transcriptional activity and expression. (A) 293T cells were co-transfected with thymidine kinase luciferase reporter with either pLVX-Tight-Puro empty vector, HA-wtE2F4, HA-E2F4(Ser) ${ }_{12}$ or HA-E2F4(Ser) ${ }_{14}$ and pLVX-Tet-Off Advanced in absence of tetracycline. pRL-SV40 Renilla luciferase reporter control vector was also co-transfected. Forty-eight hours following transfection, luciferase activity was quantified and normalized using the Renilla reporter, with the pLVX-Tight-Puro empty vector condition set at 1 . Fold induction represents the average of three separate experiments. ${ }^{* *}$ Significantly different from control $(\mathrm{p}<0.01)$. "Significantly different from control ( $p<0.05$, Student's t-test). (B and C) 293T cells were co-transfected with either pLVX-Tight-Puro empty vector, HA-wtE2F4, HA-E2F4(Ser) ${ }_{12}$ or HA-E2F4(Ser) ${ }_{14}$ and pLVX-Tet-Off Advanced in absence of tetracycline. Forty-eight hours following transfection, cells were lysed and E2F4 expression was analyzed by western blot analysis. $\beta$-actin was used as a loading control (B). Forty-eight hours following transfection, cells were processed according to the Qiagen RNeasy protocol to harvest total RNAs. E2F4 and RPLPO mRNA levels were quantified as described in Materials and methods (C).

at different time intervals in order to analyze protein expression levels of E2F4 forms. As shown in Fig. 2A (lanes 2 versus 7 and 12), expression of E2F4(Ser) ${ }_{12}$ and $\mathrm{E} 2 \mathrm{~F} 4(\mathrm{Ser})_{14}$ mutants was higher than wild-type E2F4 expression in untreated cells (time 0). Following cycloheximide treatment however, E2F4(Ser) ${ }_{12}$ and E2F4(Ser) ${ }_{14}$ protein levels decreased much more slowly than that of wild-type E2F4 (Fig. 2A and B, for densitometric analysis). Specifically, $3 \mathrm{~h}$ after cycloheximide addition, expression of E2F4 protein was drastically decreased while expression of E2F4(Ser) $)_{12}$ and E2F4(Ser) $)_{14}$ mutants remained at control (time 0) levels. Accordingly, the half-life of wild-type E2F4 protein was $\sim 5 \mathrm{~h}$ whereas E2F4(Ser) ${ }_{12}$ and E2F4(Ser) ${ }_{14}$ half-lives were $>12 \mathrm{~h}$.

Furthermore, association of E2F transcription factors with a pocket protein has been reported to protect $\mathrm{E} 2 \mathrm{~F}$ factors against degradation (20-22). We therefore analyzed whether colorectal cancer-associated mutations modulate E2F4 interaction with p130/RBL2, the main pocket protein partner for E2F4 in various cell types including intestinal epithelial cells (9). Indeed, association of E2F4 with p130 was found to promote E2F4 stability (20). Thus, cells were co-transfected with p130 along with wild-type E2F4, E2F4(Ser) ${ }_{12}$ or E2F4(Ser) ${ }_{14}$ after which E2F4 was immunoprecipitated and the amounts of associated p130 analyzed by western blotting. As shown in Fig. 2C, neither E2F4(Ser) $)_{12}$ nor E2F4(Ser) $)_{14}$ were found to have an altered capacity to associate with p130 in comparison to wild-type E2F4 (when normalized to immunoprecipitated E2F4 levels). These results suggest that the colorectal cancerassociated E2F4 mutants are more stable than wild-type E2F4, independently of their capacity to interact with p130.

Colorectal cancer cells with MSI exhibit enhanced E2F4 expression. The CAG triplet repeat in the coding region of the $E 2 F-4$ gene has been reported to be mutated in colorectal cancers exhibiting a microsatellite instability (MSI) phenotype. We therefore analyzed E2F4 gene and protein expression patterns in a panel of colorectal cancer cell lines which are microsatellite unstable (MSI) (HCT116, SW48, DLD-1, LoVo) and microsatellite stable (MSS) (COLO205, Caco-2/15 and HT-29) $(23,24)$. As shown in Fig. 3A and B, E2F4 mRNA and protein levels were observed to be globally enhanced in colorectal cancer cell lines exhibiting microsatellite instability.

Given this increased E2F4 expression in MSI colorectal cancer cells, the number of CAG repeats in the E2F4 microsatellite was determined in each cell line by capillary electrophoresis as described in Materials and methods. Analysis of the 13-serine residue microsatellite of E2F4 revealed that only the SW48 cell line expressed mutated E2F4 with a deletion of one CAG repeat causing a deletion of one serine residue. Interestingly, amongst all colorectal cancer cell lines analyzed, SW48 exhibited the highest expression of E2F4 protein (Fig. 3A). In order to associate this increased protein expression with increased E2F4 stability, endogenous E2F4 half-life was analyzed in SW48 cells comparatively to Caco-2/15, a MSS cell line in which E2F4 is wild-type. SW48 and Caco-2/15 cells were treated with cycloheximide to inhibit protein synthesis for various time intervals. As shown in Fig. 3C and D, following cycloheximide treatment, E2F4 protein levels decreased more rapidly in Caco-2/15 compared to SW48 cells. Specifically, E2F4 half-life in Caco-2/15 cells was established at $19 \mathrm{~h}$ whereas E2F4 half-life in SW48 cells was $\sim 48 \mathrm{~h}$. These results confirm that the deletion of one serine residue in the serine stretch increases E2F4 stability resulting in increased protein levels. 
A

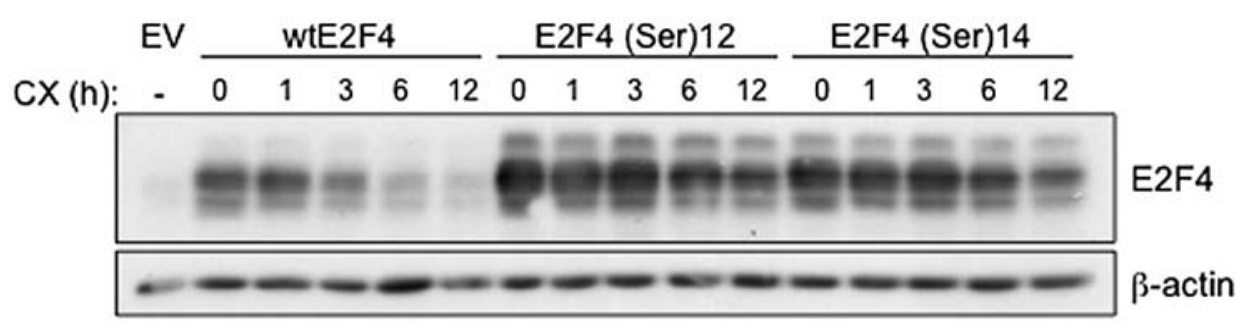

B

C $\mathrm{IP}: \mathrm{E} 2 \mathrm{~F} 4$
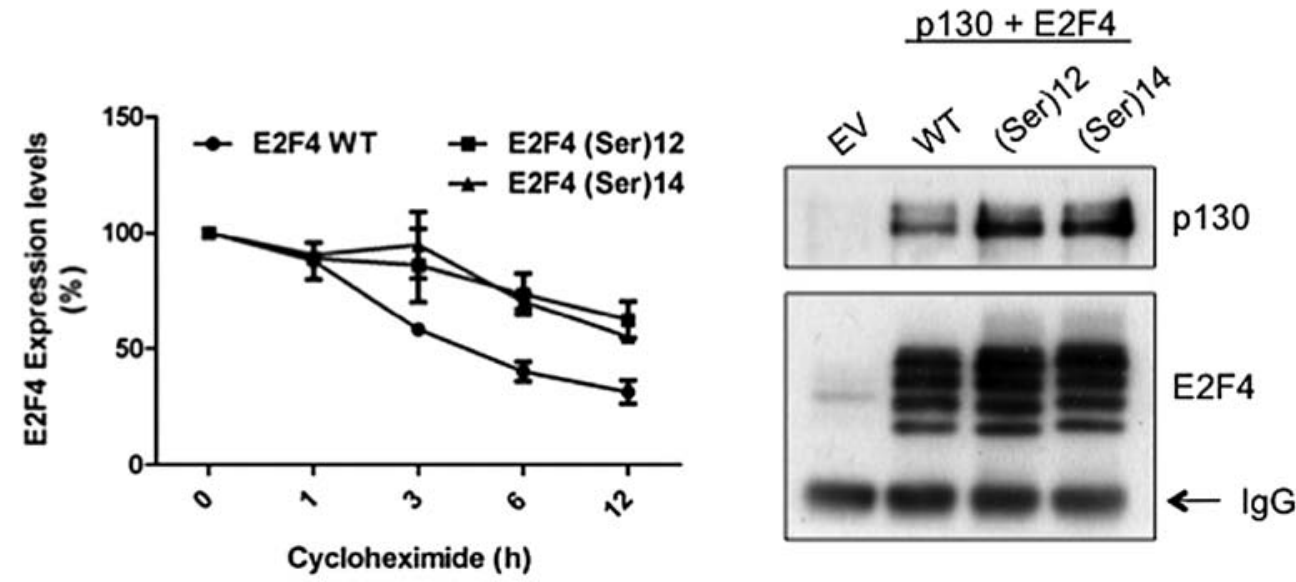

Figure 2. Colorectal cancer-associated mutations increase E2F4 protein stability. (A) Twenty-four hours following transfection, cells were treated with cycloheximide $(10 \mu \mathrm{g} / \mathrm{ml})$ and harvested after $0,1,3,6$ or $12 \mathrm{~h}$. All cells were processed and lysed at the same time. E2F4 and $\beta$-actin expression was analyzed by western blot analysis. (B) Densitometric analysis of data shown in (C) is represented. E2F4 expression at $0 \mathrm{~h}$ of cycloheximide was set at $100 \%$. Relative E2F4 expression levels were calculated using $\beta$-actin as reference. (C) 293T cells were transfected with either pLVX-Tight-Puro empty vector, HA-wtE2F4, HA-E2F4(Ser) ${ }_{12}$ or HA-E2F4(Ser) ${ }_{14}$ as well as pLVX-Tet-Off Advanced and pCDNA1-p130 in absence of tetracycline. Forty-eight hours following transfection, cells were lysed and E2F4 was immunoprecipitated from cleared lysates. E2F4 immunocomplexes were separated by SDS-PAGE gels and western blot analysis was performed using specific antibodies against E2F4 and p130. EV, empty vector; WT, wild-type; IP, immunoprecipitation.

E2F4(Ser $)_{12}$ or E2F4(Ser $)_{14}$ expression enhances the capacity of colorectal cancer cells to grow in soft agar. Our previous report indicated that $\mathrm{E} 2 \mathrm{~F} 4$ transcription factor expression is required for anchorage-dependent and anchorage-independent growth of colorectal cancer cells (8). Because anchorageindependent growth potential may better correlate with tumorigenic growth in vivo, we determined whether E2F4 mutations in the serine stretch was correlated with stimulation of tumor cell growth in soft agar. The DLD-1 cell line was chosen because this line exhibits microsatellite instability and expresses moderate levels of endogenous wild-type E2F4. As shown in Fig. 4, expression of E2F4(Ser) ${ }_{12}$ or E2F4(Ser) ${ }_{14}$ mutants (Fig. 4A) increased the capacity of DLD-1 cells to form colonies in soft agar (Fig. 4B). Moreover, colonies formed following the expression of E2F4 mutants were significantly larger than colonies formed following the expression of wildtype E2F4 (Fig. 4C). Of note, similar results were obtained with the MSS cell line Caco-2/15 (data not shown).

\section{Discussion}

Microsatellite instability is one of the molecular pathways leading to colorectal cancer progression (4). Several genes containing microsatellite and encoding regulators of cell proliferation are affected by MSI in colorectal cancer (e.g. $T G F-\beta$ type II receptor, TCF-4, activin receptor-2, insulin-like growth factor-2) (4). Among the E2F transcription factor family, E2F4 is the only member that has a 13 consecutive serine residue microsatellite in its transactivation domain (14). A number of studies report that this specific E2F4 microsatellite is frequently mutated in colorectal cancers bearing microsatellite instability and that the most common mutations observed are the addition or withdrawal of a single serine residue (10-13). These mutants show elevated transactivation of an E2F consensus promoter sequence and promote proliferation of murine fibroblasts (25). However, the mechanism and functional impact of this increased transactivation remains to be understood in the context of intestinal epithelial cells.

Herein, our results further confirm that the addition or deletion of a single serine residue in the E2F4 microsatellite increases the transactivation potential of E2F4 on a target gene that is important for cell proliferation, namely thymidine kinase. This increased transcriptional activity was also associated with increased expression of E2F4 protein. Indeed, both E2F4 mutants exhibited increased stability. This is consistent with previous results showing that deletion of the carboxy- 
A

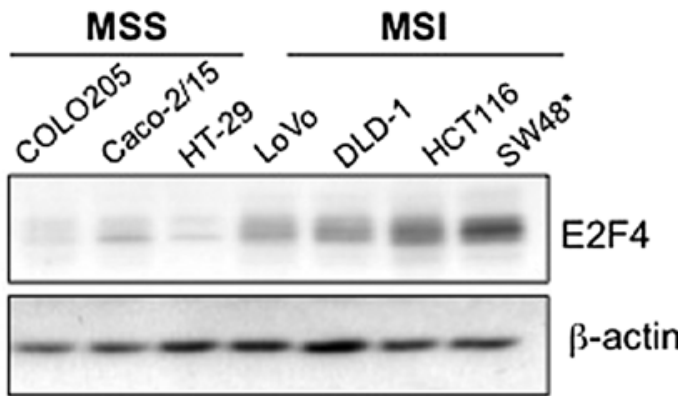

B
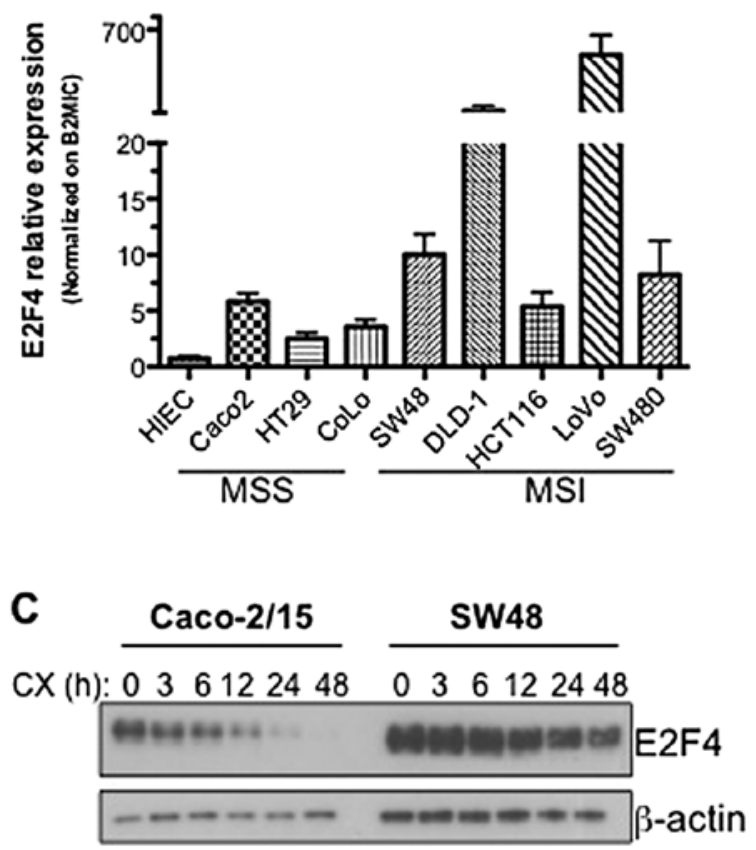

D

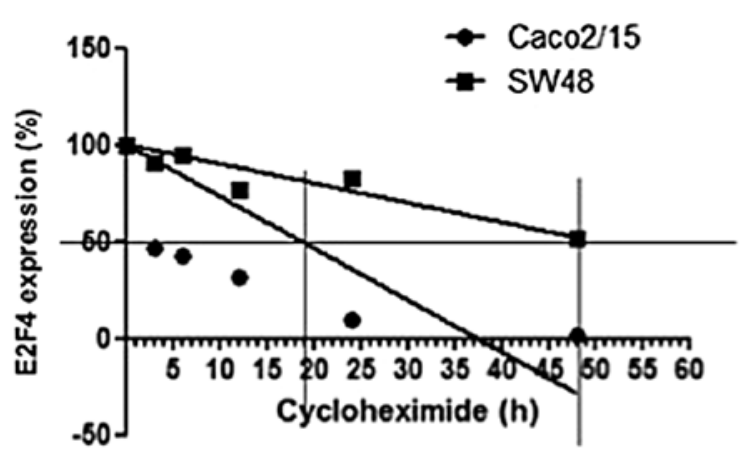

Figure 3. Colorectal cancer cells with MSI exhibit enhanced E2F4 expression. (A) E2F4 protein expression was analyzed in subconfluent growing HIEC and various colorectal cancer cell lines by western blotting using specific antibody. $\beta$-actin was used as a loading control. (B) Subconfluent HIEC and colorectal cancer cells were processed according to the Qiagen RNeasy protocol to harvest total RNAs. E2F4 and $\beta 2$-microglobulin mRNAs were quantified by real-time qPCR as described in Materials and methods. $\beta 2 \mathrm{MIC}, \beta 2$-microglobulin. (C) Caco-2/15 and SW48 cells were treated with cycloheximide $(10 \mu \mathrm{g} / \mathrm{ml})$ and harvested after $0,3,6,12,24$ or $48 \mathrm{~h}$. Cells were all processed and lysed at the same time. E2F4 and $\beta$-actin expression was analyzed by western blot analysis. (D) Densitometric analysis of data shown in (C) is presented. E2F4 expression at $0 \mathrm{~h}$ of cycloheximide was set at $100 \%$. Relative E2F4 expression levels were calculated using $\beta$-actin as reference.
A

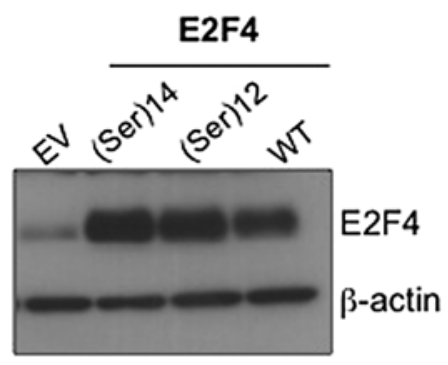

B

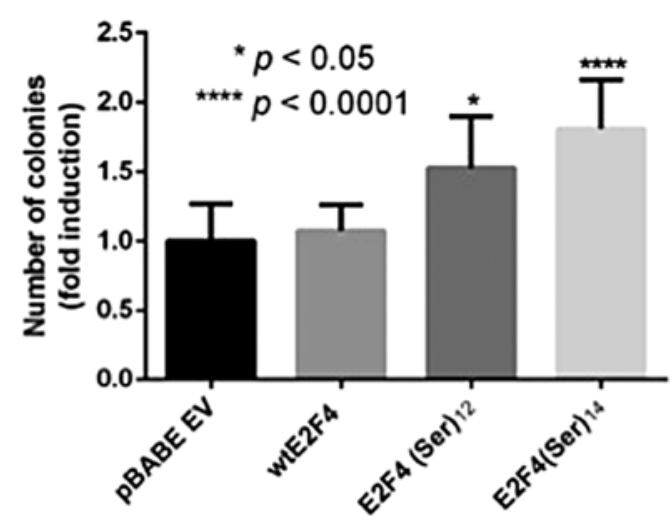

C

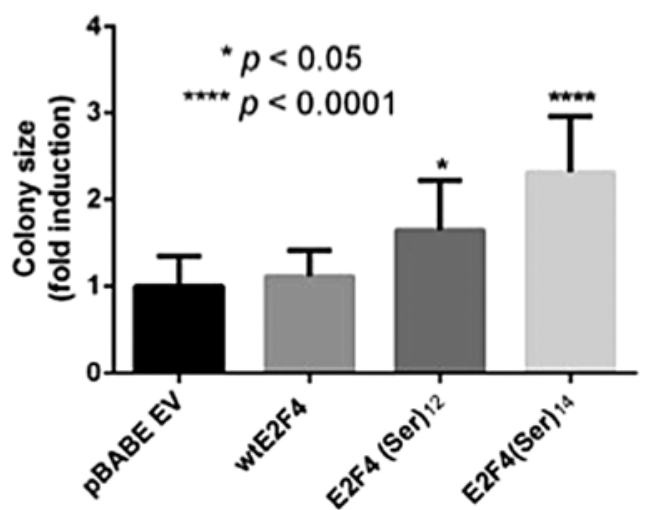

Figure 4. E2F4(Ser) $)_{12}$ or E2F4(Ser $)_{14}$ expression enhances the capacity of colorectal cancer cells to grow in soft agar. (A) DLD-1 cells were infected with either pBABE-Puro empty vector (EV), HA-wtE2F4, HA-E2F4(Ser) or HA-E2F4(Ser) ${ }_{14}$ vectors and populations were selected with puromycin $(5 \mu \mathrm{g} / \mathrm{ml})$ for 10 days. Cells were lysed and E2F4 expression was analyzed by western blot analysis. $\beta$-actin was used as a loading control. (B and C) DLD-1 cells expressing pBABE-Puro EV, HA-wtE2F4, HA-E2F4(Ser) or HA-E2F4(Ser) $)_{14}$ were seeded in growth medium containing $0.7 \%$ agarose. Results indicate the number of colonies formed (A) along with their respective sizes $(B)$. Results are the mean \pm SD of three separate experiments. "Significantly different from control (pBABE HA-wtE2F4) at $\mathrm{p}<0.05$ (Student's t-test). ${ }^{* * * * *}$ Significantly different from control (pBABE HA-wtE2F4) at $\mathrm{p}<0.0001$ (Student's t-test).

terminal 112 amino acids of E2F4 led to a dramatic increase in E2F-4 half-life. Taken together, these results suggest that the E2F4 C-terminus (which includes its transactivation domain, the pocket protein interaction domain and the 13 serine stretch) brings about instability (20). Of note, several studies have described the importance of pocket protein interaction for E2F transcription factor stability (20-22). However, our co-immunoprecipitation studies did not demonstrate any altered association of E2F4 mutants with p130, the main 
pocket protein partner of E2F4 in intestinal epithelial cells. Association with DP-2 was also analyzed given that DP-2 is known to induce E2F4 nuclear localization and could hence protect E2F4 from cytoplasmic degradation (26). However, DP-2 association with E2F4(Ser) ${ }_{12}$ or E2F4(Ser) $)_{14}$ mutants was not affected (data not shown). Interestingly, the E2F4 serine stretch comprises phosphorylation sites for GSK3 $\beta$, a kinase known to target many cell cycle regulatory proteins to the proteasome for degradation (27-29). Preliminary data obtained in our laboratory suggest that GSK $3 \beta$ can interact with and phosphorylate E2F4, at least in vitro (data not shown). Whether endogenous E2F4 is indeed a potent substrate for GSK3 $\beta$ in cells remains to be established. Further studies will be needed to firmly elucidate the molecular mechanisms underlying the increased stability of E2F4 mutants.

In the present study, we showed that both protein and mRNA expression levels of the E2F4 transcription factor were higher in CRC cell lines bearing microsatellite instability compared to both microsatellite-stable cell lines and normal intestinal epithelial cells. Of note, however, mRNA levels did not exactly follow protein levels, suggesting the implication of mechanisms other than transcription in the regulation of E2F4 protein levels in CRC cells. Endogenous sequence analysis of E2F4 in several CRC cell lines further revealed that only SW48 expressed a mutated E2F4 with 12 serines in its microsatellite. Stability analysis also confirmed that E2F4 was more stable in SW48 than in Caco-2/15 cells reinforcing the notion that E2F4 mutations in the microsatellite sequence increase E2F4 stability. Of particular interest, the increased expression of E2F4 observed in SW48 cells could contribute to their higher capacity to form tumors in nude mice compared to Caco-2/15 cells (30). Furthermore, these findings are in agreement with our results demonstrating that the expression of E2F4(Ser) $)_{12}$ or E2F4(Ser) $)_{14}$ mutants in DLD-1 cells significantly increased their capacity to grow in soft agar.

E2F transcription factors have long been known to promote transformation featuring anchorage-independent survival and growth, as well as loss of contact inhibition $(31,32)$. Accordingly, we previously reported the requirement of E2F4 for anchorage-independent growth of CRC cells (8). In addition to these observations, increased nuclear expression of E2F4 in breast cancer has been associated with poor prognosis with larger tumors, recurrent disease, distant metastasis and poorer outcome (33). Although such investigation remains to be performed for colorectal cancer, our results indicate that mutations of $\mathrm{E} 2 \mathrm{~F} 4$ promote the capacity of cancer cells to grow without anchorage, thereby contributing to tumor progression and metastasis formation (34).

We and others have previously shown that E2F4 protein levels are increased in human colorectal tumors. Since microsatellite instability is observed in $\sim 15 \%$ of colorectal cancers, other mechanisms in addition to mutations should account for the enhanced expression of E2F4. Nevertheless, our data demonstrate that, when mutated in its serine stretch, E2F4 protein is more stable, more readily expressed and more transcriptionally active. As a result, colorectal cancer-associated E2F4 mutations may confer increased tumorigenic potential for tumors with microsatellite instability, thus providing further insight into the functional impact of these mutations in CRC.

\section{Acknowledgements}

We thank Pierre Pothier for the critical reading of the manuscript. We also thank Anne Vézina for technical assistance. We gratefully acknowledge the work of Mathieu Durand, research assistant at the Laboratoire de Génomique Fonctionnelle de l'Université de Sherbrooke (LGFUS) for the determination of E2F4 microsatellite mutations in colorectal cancer cell lines. This study was supported by a grant from the Cancer Research Society Inc. and from the Canadian Institutes of Health Research MT-14405 (to N.R.). Marie-Christine Paquin is a recipient of a NSERC fellowship. Nathalie Rivard is a recipient of a Canadian Research Chair in Colorectal Cancer and Inflammatory Cell Signaling and a member of the FRSQfunded 'Centre de Recherche Clinique Étienne LeBel'.

\section{References}

1. Yang VW, Lewis J, Wang TC and Rustgi AK: Colon cancer: an update and future directions. Gastroenterology 138: 2027-2028, 2010.

2. van der Flier LG and Clevers H: Stem cells, self-renewal, and differentiation in the intestinal epithelium. Annu Rev Physiol 71: 241-260, 2009.

3. Worthley DL and Leggett BA: Colorectal cancer: molecular features and clinical opportunities. Clin Biochem Rev 31: 31-38, 2010.

4. Boland CR and Goel A: Microsatellite instability in colorectal cancer. Gastroenterology 138: 2073-2087.e3, 2010.

5. Tsantoulis PK and Gorgoulis VG: Involvement of E2F transcription factor family in cancer. Eur J Cancer 41: 2403-2414, 2005.

6. Chen HZ, Tsai SY and Leone G: Emerging roles of E2Fs in cancer: an exit from cell cycle control. Nat Rev Cancer 9: 785-797, 2009

7. Gaubatz S, Lindeman GJ, Ishida S, Jakoi L, Nevins JR, Livingston DM and Rempel RE: E2F4 and E2F5 play an essential role in pocket protein-mediated G1 control. Mol Cell 6: 729-735, 2000.

8. Garneau H, Paquin MC, Carrier JC and Rivard N: E2F4 expression is required for cell cycle progression of normal intestinal crypt cells and colorectal cancer cells. J Cell Physiol 221: 350-358, 2009.

9. Deschenes C, Alvarez L, Lizotte ME, Vezina A and Rivard N: The nucleocytoplasmic shuttling of E2F4 is involved in the regulation of human intestinal epithelial cell proliferation and differentiation. J Cell Physiol 199: 262-273, 2004.

10. Yoshitaka T, Matsubara N, Ikeda M, Tanino M, Hanafusa H, Tanaka N and Shimizu K: Mutations of E2F-4 trinucleotide repeats in colorectal cancer with microsatellite instability. Biochem Biophys Res Commun 227: 553-557, 1996.

11. Souza RF, Yin J, Smolinski KN, et al: Frequent mutation of the E2F-4 cell cycle gene in primary human gastrointestinal tumors. Cancer Res 57: 2350-2353, 1997.

12. Ikeda M, Orimo H, Moriyama $\mathrm{H}$, et al: Close correlation between mutations of E2F4 and hMSH3 genes in colorectal cancers with microsatellite instability. Cancer Res 58: 594-598, 1998.

13. Moriyama H, Sasamoto H, Kambara T, et al: E2F-4 mutation in hereditary non-polyposis colorectal cancer. J Exp Clin Cancer Res 21: 185-189, 2002.

14. Sardet C, Vidal M, Cobrinik D, Geng Y, Onufryk C, Chen A and Weinberg RA: E2F-4 and E2F-5, two members of the E2F family, are expressed in the early phases of the cell cycle. Proc Natl Acad Sci USA 92: 2403-2407, 1995.

15. Wu CL, Zukerberg LR, Ngwu C, Harlow E and Lees JA: In vivo association of E2F and DP family proteins. Mol Cell Biol 15: 2536-2546, 1995.

16. Perreault $\mathrm{N}$ and Beaulieu JF: Use of the dissociating enzyme thermolysin to generate viable human normal intestinal epithelial cell cultures. Exp Cell Res 224: 354-364, 1996.

17. Dou QP, Zhao S, Levin AH, Wang J, Helin K and Pardee AB: G1/S-regulated E2F-containing protein complexes bind to the mouse thymidine kinase gene promoter. J Biol Chem 269: 1306-1313, 1994. 
18. Jansen-Durr P, Meichle A, Steiner P, et al: Differential modulation of cyclin gene expression by MYC. Proc Natl Acad Sci USA 90: 3685-3689, 1993.

19. Oswald F, Lovec H, Moroy T and Lipp M: E2F-dependent regulation of human MYC: Trans-activation by cyclins D1 and A overrides tumour suppressor protein functions. Oncogene 9: 2029-2036, 1994.

20. Hateboer G, Kerkhoven RM, Shvarts A, Bernards R and Beijersbergen RL: Degradation of E2F by the ubiquitin-proteasome pathway: Regulation by retinoblastoma family proteins and adenovirus transforming proteins. Genes Dev 10: 2960-2970, 1996.

21. Hofmann F, Martelli F, Livingston DM and Wang Z: The retinoblastoma gene product protects E2F-1 from degradation by the ubiquitin-proteasome pathway. Genes Dev 10: 2949-2959, 1996.

22. Martelli F and Livingston DM: Regulation of endogenous E2F1 stability by the retinoblastoma family proteins. Proc Natl Acad Sci USA 96: 2858-2863, 1999.

23. Heinen CD, Richardson D, White R and Groden J: Microsatellite instability in colorectal adenocarcinoma cell lines that have full-length adenomatous polyposis coli protein. Cancer Res 55: 4797-4799, 1995.

24. Boyer JC, Umar A, Risinger JI, et al: Microsatellite instability, mismatch repair deficiency, and genetic defects in human cancer cell lines. Cancer Res 55: 6063-6070, 1995.

25. Takashima H, Matsumoto Y, Matsubara N, et al: Effect of naturally occurring E2F-4 alterations on transcriptional activation and proliferation in transfected cells. Lab Invest 81: 1565-1573, 2001.
26. Martelli F, Hamilton T, Silver DP, et al: p19ARF targets certain E2F species for degradation. Proc Natl Acad Sci USA 98: 4455-4460, 2001.

27. Vlach J, Hennecke S and Amati B: Phosphorylation-dependent degradation of the cyclin-dependent kinase inhibitor p27. EMBO J 16: 5334-5344, 1997.

28. Diehl JA, Cheng M, Roussel MF and Sherr CJ: Glycogen synthase kinase-3beta regulates cyclin D1 proteolysis and subcellular localization. Genes Dev 12: 3499-3511, 1998.

29. Vandel L and Kouzarides T: Residues phosphorylated by TFIIH are required for E2F-1 degradation during S-phase. EMBO J 18: 4280-4291, 1999.

30. Dunn EF, Iida M, Myers RA, et al: Dasatinib sensitizes KRAS mutant colorectal tumors to cetuximab. Oncogene 30: 561-574, 2011.

31. Chen C and Wells AD: Comparative analysis of E2F family member oncogenic activity. PLoS One 2: e912, 2007.

32. Xu G, Livingston DM and Krek W: Multiple members of the E2F transcription factor family are the products of oncogenes. Proc Natl Acad Sci USA 92: 1357-1361, 1995.

33. Rakha EA, Pinder SE, Paish EC, Robertson JF and Ellis IO: Expression of E2F-4 in invasive breast carcinomas is associated with poor prognosis. J Pathol 203: 754-761, 2004.

34. Mori S, Chang JT, Andrechek ER, et al: Anchorage-independent cell growth signature identifies tumors with metastatic potential. Oncogene 28: 2796-2805, 2009. 